\title{
A microrheological study of hydrogel kinetics and micro-heterogeneity
}

\author{
Anders Aufderhorst-Roberts ${ }^{1, a}$, William J. Frith ${ }^{2}$, and Athene M. Donald ${ }^{1}$ \\ 1 Cavendish Laboratory, Department of Physics, University of Cambridge, JJ Thomson Avenue, Cambridge, CB3 0HE, UK \\ 2 Unilever R\&D Colworth, Colworth Science Park, Sharnbrook, Bedford, MK44 1LQ, UK
}

Received 19 October 2013 and Received in final form 31 January 2014

Published online: 27 May 2014

(C) The Author(s) 2014. This article is published with open access at Springerlink.com

\begin{abstract}
The real-time dynamic heterogeneity of the gelation process of the amino acid derivative Fmoctyrosine (Fmoc-Y) is studied using particle tracking microrheology. To trigger gelation, glucono- $\delta$-lactone $(\mathrm{GdL})$ is added, which gradually lowers the $p \mathrm{H}$ over several hours. The onset of self-assembly in the system is signified by a sharp drop in the mean-squared displacement of embedded particles, a phenomenon that is found to correlate with the $p \mathrm{H}$ of the system reaching the $p K_{a}$ of Fmoc-Y. The gel point is identified and found to be dependent on the GdL concentration. Analysis of embedded probe particle dynamics allows the heterogeneity of the sample to be quantified, using three metrics: the heterogeneity ratio (HR), the non-Gaussian parameter of the van Hove correlation function $(N)$ and the bin distribution of the meansquared displacement (MSD) of single particles $(f(z))$. Results from the three techniques are found to be approximately comparable, with increases in heterogeneity observed in all samples for incubation times $t_{w}=0-3$ hours. The final heterogeneity in all samples is found to be remarkably low compared to other systems previously reported in the literature.
\end{abstract}

\section{Introduction}

Hydrogels are a highly versatile class of materials with potential applications in wound treatment [1], tissue engineering [2] and drug delivery [3]. One strategy for preparing hydrogels with controllable properties is through the self-assembly of small molecules commonly known as lowmolecular-weight hydrogelators [4]. Such molecules are advantageous due to their accessibility, low cost and potential for modification, as well as their often unique mechanical properties [5].

The range of available low-molecular-weight hydrogelating systems has increased in recent years as their discovery has steadily relied more upon rational design and less upon serendipity [6]. Hydrogelating systems that have been amongst the most extensively studied include peptides which adopt a $\beta$-hairpin conformation [7], peptide amphiphiles [8] and $\alpha$-helical coiled-coil systems [9]. Much attention has also been given to aromatic peptide-conjugate gelators such as peptides capped with pyrene [10] and naphthalene [11]. By far the most studied group of aromatic peptide conjugates are peptides capped with the fluorenylmethyloxycarbonyl moiety

a Present address: School of Physics and Astronomy, E. C. Stoner building, University of Leeds, Woodhouse Lane, Leeds, LS2 9JT, UK. e-mail: A.Aufderhorst-Roberts@leeds.ac.uk
(Fmoc) [12]. Hydrogels can also be formed from Fmocamino acids, with Fmoc-Tyrosine being the most commonly studied [13-16]. Here, gelation can be brought about by either lowering the $p \mathrm{H}$ of the system [16] or by enzymatically dephosphorylating Fmoc-Y-phosphate [13]. One novel approach is to lower the $p \mathrm{H}$ gradually using glucono- $\delta$-lactone (GdL), which hydrolyses to gluconic acid resulting in a gradual decrease in $p \mathrm{H}$ and a final gel that is transparent [17]. The transparency of the gel suggests a relatively homogeneous microstructure but the heterogeneity has yet to be measured quantitatively.

Important factors in designing hydrogels for specific applications include the kinetics of formation [18] and the spatial heterogeneity of the hydrogel network [19]. Understanding the former is essential when designing socalled smart systems that gel under pre-specified conditions [20], whereas the latter is highly relevant to a number of biological applications. For example, recent studies have identified the importance of micro-heterogeneity in the functionality of the extra-cellular matrix [21] and in the vulnerability of healthy tissue to invasion from cancerous cells [22]. Micro-heterogeneity is also an important factor in the function and adaptability of naturally occurring materials, such as bacterial biofilms, making it a subject of wider biological interest [23].

Passive microrheology is a group of techniques developed over recent years, which measure the thermal 
fluctuations of micron-scale probe particles embedded in a sample, thereby allowing the micro-scale viscoelastic [24], kinetic [25] and structural [19] properties of a sample material to be inferred. It therefore presents an attractive strategy for measuring the self-assembly properties of low-molecular-weight hydrogelators. Gelation kinetics can be investigated through examining how the meansquared displacements (MSD) of particles with respect to lag time change as the sample evolves. Similarly microheterogeneity can be quantified by comparing the trajectories of individual probe particles. This is a unique advantage of microrheology over bulk rheology as such information cannot be extracted from bulk rheological data as the length scales studied are much larger [26]. Early work on using microrheology to probe heterogeneity focussed on biologically relevant materials such as actin, both as filaments and bundles [27] and as $\alpha$-actinin cross-linked networks [28]. There are also reports of microrheological studies of the heterogeneity of solutions of chromosonal DNA [29] and of industrially relevant materials such as Laponite [18] and wheat gliadin suspensions [30]. A number of different metrics have been developed by various research groups to quantify micro-heterogeneity [31-33], although few studies have compared these different approaches. In addition, the majority of applications of these metrics have been to materials with relatively high heterogeneity and it is unclear how applicable they are to materials with lower heterogeneity.

In this study the micro-heterogeneity and gelation kinetics of Fmoc-Tyrosine (Fmoc-Y), a low-molecularweight hydrogelator, are examined. Specifically the link between changes in $p \mathrm{H}$ and in microstructure are explored, such as the onset of gelation through percolation of aggregates in solution as well as the micro-heterogeneity of the hydrogel network over time. Fmoc-Y has been observed to form particularly clear (and therefore potentially homogeneous on the micro-scale) hydrogels when gelled using a range of different concentrations of GdL [34]. For this study concentrations of $\mathrm{GdL}$ are selected so that gelation occurs over several hours, thereby allowing ample data to be collected both before and after the sol-gel transition. The gradual nature of gelation also ensures that the sample does not evolve during the course of a single microrheology measurement. Varying the GdL concentration varies the $p \mathrm{H}$ and thus also allows the $p \mathrm{H}$ sensitivity of the gel's structural properties to be investigated. Values of three different metrics that have previously been reported are compared and contrasted to comprehensively assess the heterogeneity. These are the heterogeneity ratio (HR) [33], the non-Gaussian parameter of the van Hove correlation function $(N)$ [32] and the bin distribution of the single particle MSD $(f(z))$ [31]. The narrow range of GdL concentrations means that the change in each of these metrics is likely to be subtle. To contextualise the magnitudes of these metrics they are therefore also compared to previously published values of other systems. Applying the metrics before and after the sol-gel transition also allows their applicability to more viscous samples (pre-gelation) and more elastic samples (post-gelation) to be critically assessed. Furthermore Fmoc-Y has previously been reported to form a gel with modulus $G^{\prime} \approx 2000 \mathrm{~Pa}$ at a time 20 hours [16]. Particle tracking microrheology cannot be reliably applied to such stiff samples as the largest moduli that can accurately be measured are limited by the smallest measureable amplitude of particle fluctuations. Thus particle tracking microrheology is typically not effective for materials with $G^{\prime} \gtrsim 1 \mathrm{~Pa}[26]$ and measurements in this study can not be extended to long incubation times.

\section{Materials and methods}

\subsection{Materials}

All materials are purchased from Sigma-Aldrich and Millipore water is used throughout.

\subsection{Sample preparation}

For preparation of Fmoc-Y hydrogels, Fmoc-Y-OH (42 mg, $0.104 \mathrm{mmol}$ ) is dissolved in $10 \mathrm{ml}$ sodium hydroxide $(0.0004 \%$ weight/volume, $0.1 \mathrm{mmol})$, resulting in the formation of a salt, Fmoc-Y-ONa (see fig. 1(a)) in an aqueous solution. The solution is gently warmed to $40^{\circ} \mathrm{C}$ and agitated. Agitation and dissolution are repeated until the solution reaches $p \mathrm{H} 8$ (representing $10^{-6} \mathrm{M}$ unreacted $\mathrm{NaOH}$ ). Due to the inherently stochastic nature of the gelation process [35] and the well-documented instability of Fmoc groups under alkaline conditions [36], care must be taken to ensure that samples are prepared at precisely this $p \mathrm{H}$. The excess untreated Fmoc-Y-OH is removed using a syringe filter with a $220 \mathrm{~nm}$ porous membrane to leave a clear solution.

To initiate gelation, a $3 \mathrm{ml}$ aliquot of this solution is then added to GdL powder. Fmoc-Y has been found to form hydrogels over a range of GdL concentrations (5-100 mM GdL for $10 \mathrm{mM}$ Fmoc-Y) [34], however, to allow time for sufficient data before and after gelation to be collected, sample compositions are selected which form hydrogels over approximately 1-2 hours. The selected masses of $\mathrm{GdL}$ are $5.34 \mathrm{mg}, 4.01 \mathrm{mg}$ and $2.67 \mathrm{mg}$; equivalent to $10 \mathrm{mM}, 7.5 \mathrm{mM}$ and $5 \mathrm{mM} \mathrm{GdL}$, respectively. The sample is then mixed, by shaking vigorously for $20 \mathrm{~s}$. The resulting hydrolysis of GdL leads to the formation of gluconic acid via a series of intermediate steps [37]. Hence, the $p \mathrm{H}$ decreases gradually and gelation occurs over time. The molecular structure of GdL and its transition to gluconic acid is shown in fig. 1(b).

\section{$2.3 \mathrm{pH}$ measurements}

A Thermoscientific Orion 3 star bench-top $p \mathrm{H}$ meter equipped with a Fisher scientific $p \mathrm{H}$-probe is used to measure $p \mathrm{H}$. The $p \mathrm{H}$ meter is connected via a serial cable to a $\mathrm{PC}$, allowing the $p \mathrm{H}$ to be recorded every $10 \mathrm{~s}$. The $p \mathrm{H}-$ meter is calibrated before each experiment to check the response of the electrode with two buffer solutions (borate $p \mathrm{H} 10$ and phosphate $p \mathrm{H}$ 7). 


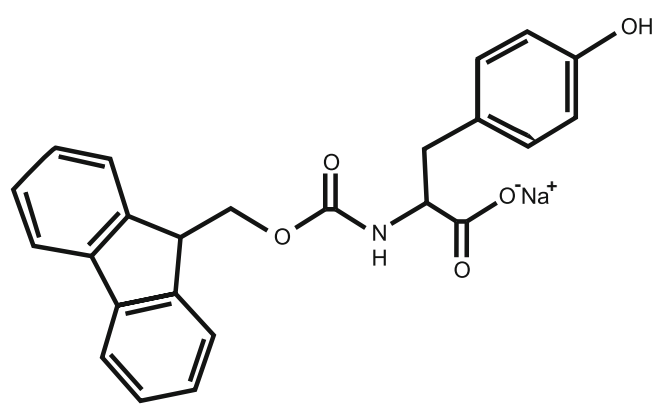

(a)

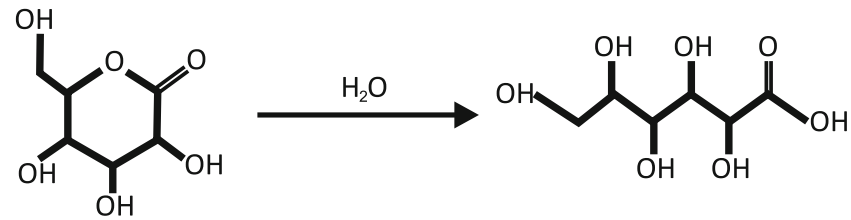

(b)

Fig. 1. The salt form of Fmoc-Y, Fmoc-Y-ONa, is used in this study because is significantly more soluble in water than its acid form. (a) Adding Glucono- $\delta$-Lactone (GdL) to a solution of Fmoc-Y-ONa. GdL hydrolyses through a series of complex chemical equilibria to form gluconic acid (b), thereby lowering $p \mathrm{H}$ gradually.

\subsection{Particle tracking}

Following sample preparation, $0.46 \mu \mathrm{m}$ latex polystyrene particles are immediately added to the sample such that their volume fraction is approximately $0.01 \%$. All particles have unmodified surface chemistry and therefore negligible surface charge. Particles are purchased from Sigma Aldrich.

Following the addition of probe particles, a $40 \mu \mathrm{l}$ aliquot of the sample is added to a sample cell (designed by Corrigan [38]), comprising a two-piece aluminium shell, which holds an o-ring sandwiched between two borosilicate glass cover slips. This design ensures that the sample is airtight and isolated from ambient thermal fluctuations. Sample temperature is maintained at $25^{\circ}$ throughout by use of a Peltier chip heat stage.

The Brownian motion of probe particles is observed using a Zeiss Axioplan LSM 500 Confocal Microscope and video clips are captured using an AVT Pike CCD camera. As gelation occurs, the displacements of embedded probe particles are expected to decrease and thus progressively higher magnification (from $32 \times$ to $50 \times$ ) and lower video capture frame rates (from $120 \mathrm{~s}^{-1}$ to $30 \mathrm{~s}^{-1}$ ) are used. The CCD camera's shutter speed is kept above the frame rate to ensure that the particle movement between frames is as low as possible. Videos are captured in a lossless avi format using AVT Amcap (part of AVT Direct Stream Package) and converted into a series of bitmap images using VirtualDub, a free video processing software package. Image analysis is carried out using in-house custom-written Matlab scripts [39].

Particle positions in individual frames are identified using a standard computational process for edge detection in images known as "Laplacian-of-Gaussian operation" [40]. This involves smoothing the image by using a Gaussian filter and then applying a Laplacian filter to detect particle edges. Background noise in the images is then reduced by systematically removing pixels whose intensity is below a defined intensity threshold. Finally, particle centroid positions are calculated by applying a double-quadratic fit. The precise position of each particle is automatically calculated frame by frame to determine an ensemble of particle trajectories. Particle trajectories which persist for shorter periods than $0.5 \mathrm{~s}$ are discounted to eliminate any remaining background noise such as speckle effects or dust on the microscope objective. From the particle trajectories, the two-dimensional mean-squared displacement (MSD) is calculated and analysed further.

Theoretically, the ensemble-averaged mean of the particle displacements caused by Brownian motion can be expected to be negligible. A significant ensemble-averaged particle displacement indicates bulk drift caused by external vibrations, temperature convection or bulk relaxation in the sample. Therefore, particle displacements are monitored over 100-frame increments and any particle movement that is common to the ensemble of particles is manually subtracted to correct for bulk drift. A further potential drawback to particle tracking is that the resolution of the tracking is limited [41] by a so-called static error which manifests itself as a constant additive term in the MSD [42]. The static error has been shown to be approximately equal to the value of the $y$-intercept of a linearly-fitted to a plot of MSD vs. lag time $\tau$ [43]. For the experiments presented here the static error is found, through this method, to be several orders of magnitude lower that the measured MSD over all incubation times $t_{w}$ and can thus be said to have a negligible effect on measurements of MSD.

\section{Results and discussion}

\subsection{Evolution of $\mathrm{pH}$}

A number of studies have highlighted the non-monotonic nature of the evolution in $p \mathrm{H}$ when $\mathrm{GdL}$ is added to Fmoc$\mathrm{Y}$ [44] or to analogous systems [35]. Such molecules are believed to have different $p K_{a}$ 's in their self-assembled form than when in solution, leading to a so-called $p K_{a}$ shift as self-assembly occurs, which manifests itself as a brief rise in $p \mathrm{H}$. A recent paper by the authors [45] has confirmed that this rise correlates approximately with the apparent onset of self-assembly, as measured through observing an initial drop in MSD, indicating an increase in viscosity. Figure 2 shows the evolution of $p \mathrm{H}$ with incubation time, 


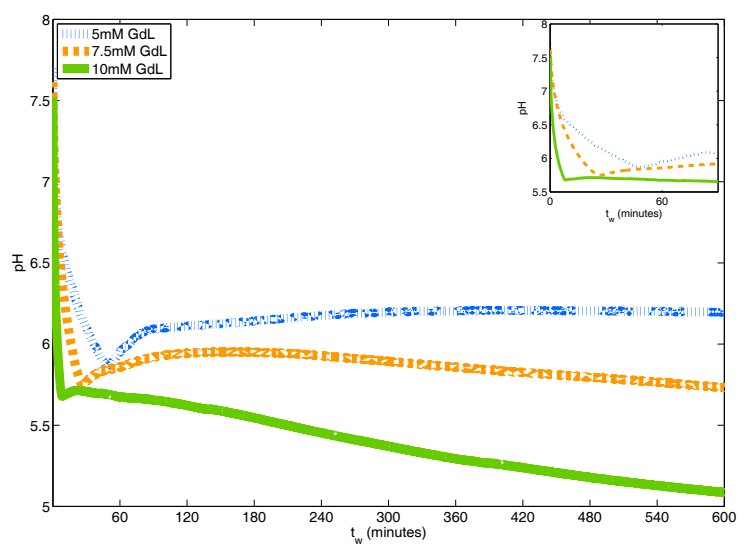

Fig. 2. $p \mathrm{H}$ vs. incubation time $t_{w}$ for a hydrogel sample prepared using $10 \mathrm{mM}$ Fmoc-Y and variable concentrations of GdL. The rate of decrease in $p \mathrm{H}$ with respect to $t_{w}$ is rapid initially and slower at longer incubation times. At relatively early incubation times the $p \mathrm{H}$ rises briefly before dropping again. Previous studies indicate that this phenomenon is linked to the onset of self-assembly [45]. Increasing the concentration of GdL appears to accelerate the rate at which $p \mathrm{H}$ decreases and results in a lower final $p \mathrm{H}$. The inset figure shows a magnified version of the $p \mathrm{H}$ at early $t_{w}$.

$t_{w}$ for samples prepared from Fmoc-Y and the three concentrations of GdL: $5 \mathrm{mM}, 7.5 \mathrm{mM}$ and $10 \mathrm{mM}$.

Figure 2 shows that the incubation time $t_{w}$ at which the $p K_{a}$ shift occurs (shown by a brief rise in $p \mathrm{H}$ ) varies with GdL concentration, suggesting that the kinetics of self-assembly are accelerated by increasing the GdL concentration. The $p \mathrm{H}$ values of the samples at later $t_{w}$ show that the eventual $p \mathrm{H}$ of the system is also dependent on GdL concentration, such that higher GdL concentrations result in lower $p \mathrm{H}$. However, the value of $t_{w}$ at which the $p K_{a}$ shift occurs is found to vary between runs and, by averaging over repeat measurements, is found to be $63.1 \pm 15.7,31.4 \pm 12.8$ and $16.6 \pm 11.2$ minutes for $5 \mathrm{mM}$, $7.5 \mathrm{mM}$ and $10 \mathrm{mM} \mathrm{GdL}$, respectively.

\subsection{Evolution of MSD}

Figure 3 shows the ensemble-averaged MSD with respect to lag time $\tau$, at selected incubation times for samples prepared using the three different GdL concentrations.

The same characteristic pattern by which plots of MSD evolve with incubation time is present for each of the three samples. For each sample, at early incubation time, the MSD exhibits power law behaviour with an exponent of 1 , which is indicative of a Newtonian fluid. At later times the curves shift downwards, indicating an increase in viscosity. At some later incubation time the logarithmic gradient decreases with $\tau$, indicating that the embedded particles are subdiffusive and that the sample has transitioned to the gel state. This indicates that the self-assembled aggregates form an infinitely connected network and is commonly referred to as the gel point $t_{\text {gel }}$ [46]. Examination of successive plots of MSD vs. $\tau$ yields values of $t_{\text {gel }}$ of $\approx 80-85$
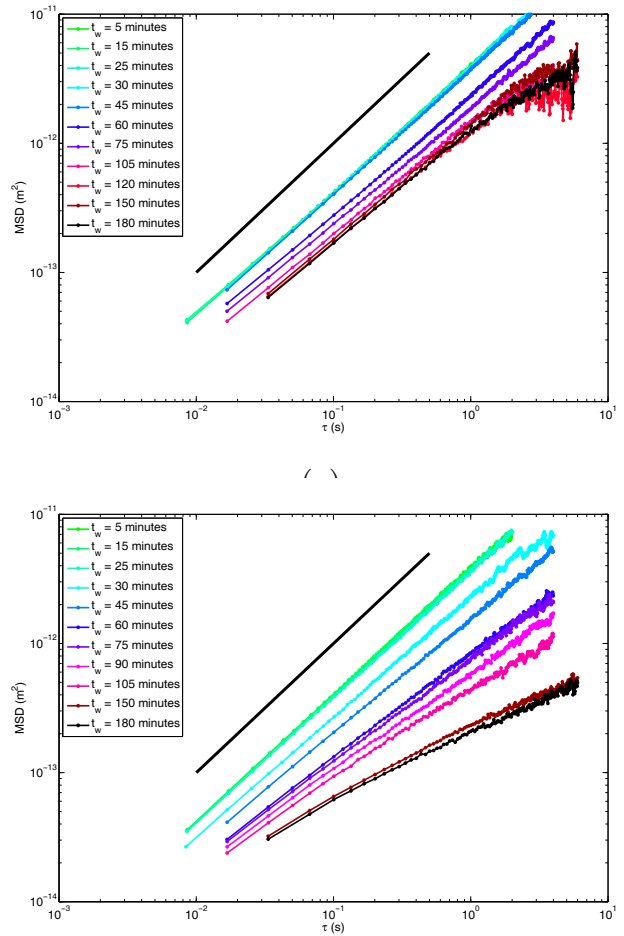

(1.)

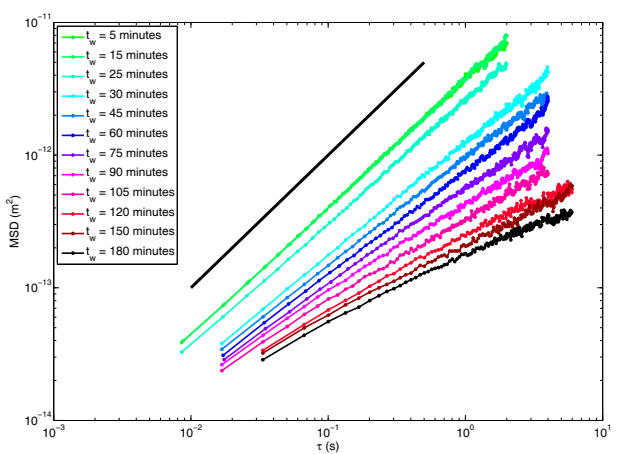

(c)

Fig. 3. Selected plots of MSD with respect to lag time $\tau$, at selected incubation times $t_{w}$ for a hydrogel sample prepared from $10 \mathrm{mM}$ Fmoc-Y and GdL concentrations of $5 \mathrm{mM}$ (a), $7.5 \mathrm{mM}$ (b) and $10 \mathrm{mM}$ (c). The pattern by which MSD evolves with respect to $t_{w}$ is similar across all three samples: at early values of $t_{w}$, the MSD indicates diffusive behaviour (the logarithmic gradient of MSD vs. $\tau$ is close to 1 ), while at later $t_{w}$, sub-diffusive behaviour is evident (the logarithmic gradient decreases with $\tau)$. From the final MSD shown $\left(t_{w}=180\right.$ minutes), it is clear that the magnitude of the MSD over all values of $\tau$ decreases as the GdL concentration is lowered, indicating that higher GdL concentrations result in gels that limit particle movement more and are therefore more robust. The solid black line indicates a power law exponent of 1 .

minutes, $\approx 55-60$ minutes and $\approx 50-55$ minutes for samples prepared using GdL concentrations of $5 \mathrm{mM}, 7.5 \mathrm{mM}$ and $10 \mathrm{mM}$ respectively. The value of $t_{\text {gel }}$ can therefore be said to be dependent on GdL concentration, such that lowering the $\mathrm{GdL}$ concentration brings about gelation at longer time scales. 


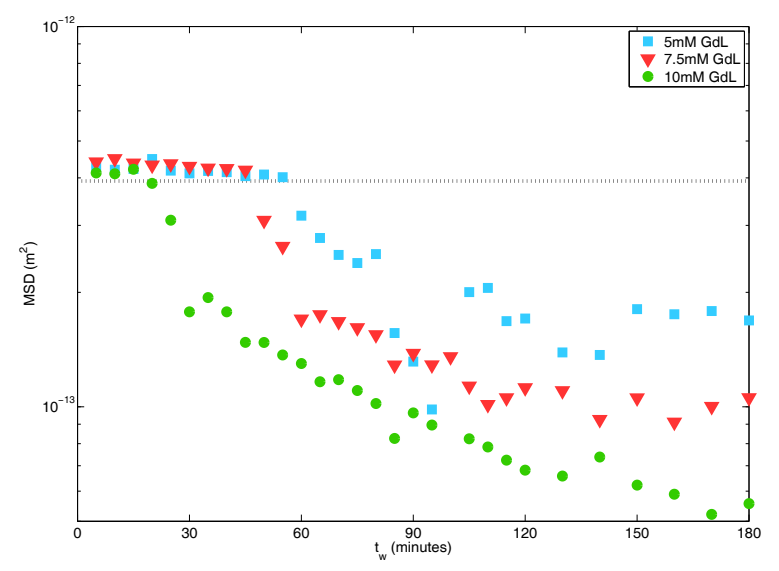

Fig. 4. MSD vs. incubation time $t_{w}$ at lag time $\tau=0.1 \mathrm{~s}$ for samples prepared using $10 \mathrm{mM}$ Fmoc-Y and varying GdL concentration. The black line shows the expected MSD for particles in water (viscosity, $\eta=8.9 \times 10^{-4}$ ). MSD is invariant over early $t_{w}$ and begins to drop at later $t_{w}$. The incubation time at which this drop occurs is later for lower GdL concentrations. Lower GdL concentrations also result in fluctuations in the MSD as it decreases. One explanation for this behaviour is that bonds between the fibres in the hydrogel are able to spontaneously form and break over time.

The difference in the magnitude of the final MSD (at $t_{w}=3$ hours) varies according to GdL concentration. Samples prepared from $5 \mathrm{mM}, 7.5 \mathrm{mM}$ and $10 \mathrm{mM}$ GdL each appear to approach a plateau in $M S D$ at later $\tau$ of: $3.002 \pm 0.492,1.353 \pm 0.384,0.415 \pm 0.0439 \times 10^{-12} \mathrm{~m}^{2}$ respectively. To quantify the hydrogel stiffness, the storage modulus $G^{\prime}$ can be approximated by [47]

$$
G^{\prime} \approx 2 K_{B} T / 3 \pi a\left\langle\Delta r^{2}\right\rangle,
$$

giving $G^{\prime}=0.00121,0.00269$ and $0.0829 \mathrm{~Pa}$ for $5 \mathrm{mM}$, $7.5 \mathrm{mM}$ and $10 \mathrm{mM}$ GdL respectively. The latter, when compared to previously published values of $G^{\prime}$ under the same conditions and time scales $\left(G^{\prime} \approx 10^{-1} \mathrm{~Pa}[45]\right)$, indicates a reasonable degree of reproducibility between samples. It should be noted that the MSD is not observed to plateau over all values of $\tau$. This, coupled with the very approximate nature of eq. (1) implies that these values of $G^{\prime}$ are highly approximate. Nevertheless, comparing these approximate values of $G^{\prime}$ over the different GdL concentrations, suggests that lowering the concentration of $\mathrm{GdL}$ results in samples that are less mechanically robust. It should be noted that recent studies on analogous molecules (naphthalene-capped dipeptides, gelled using GdL) showed the modulus of the final gel to be dependent on the kinetics of gelation but not explicitly on GdL concentration, as tuning the gelation kinetics by varying the temperature resulted in similar final gel moduli [48]. This recent finding therefore suggests that the observed differences in moduli here might be the result of the different kinetics of gelation.

The variation of MSD at $\tau=0.1 \mathrm{~s}$ with respect to $t_{w}$ for each of the samples is shown in fig. 4. For each

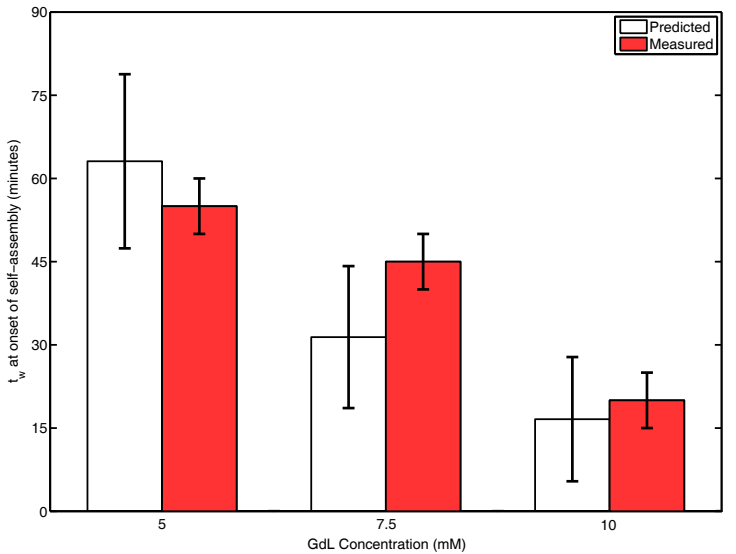

Fig. 5. Value of $t_{w}$ at the onset of self-assembly as predicted by the $p K_{a}$ shift and as measured by monitoring a drop in the MSD of embedded particles for samples prepared using various concentrations of GdL. Allowing for errors from repeat measurements, predicted and measured values of $t_{w}$ at the onset of self-assembly are in agreement.

sample, the MSD remains invariant at early $t_{w}$. In each case the MSD begins to drop abruptly at a given value of $t_{w}$, indicating the onset of self-assembly. The magnitude of the MSD for all lag times fluctuates over the range of $t_{w}$ shown. This suggests that the sol-gel transition is not instantaneous and that the system fluctuates between a liquid-like and a gel-like state, an observation that hints at the possibility of unstable bonds between aggregates in the hydrogel network forming and breaking spontaneously. It has previously been shown that the peptide derivative Fmoc-Y and related systems have final properties that depend on the choice of preparation protocol [49]. The demonstrated fluctuation in MSD with incubation time shows the microstructural properties to be highly variable with incubation time. The authors postulate that the two phenomena may be linked: variability at early incubation times through weak bonds in the hydrogel network could suggest a mechanism for changes in final rheological properties when the preparation protocols are subtly different.

The value of $t_{w}$ at which the MSD begins to drop varies with GdL concentration such that samples prepared using lower concentrations of GdL appear to begin to selfassemble at later incubation times. The predicted onset of self-assembly calculated from the $p K_{a}$ shifts (fig. 2) correlates relatively well with this measured onset of selfassembly for all samples as shown in fig. 5 .

\subsection{Micro-heterogeneity}

A very basic and approximate method for using particle tracking to assess whether a hydrogelating system is heterogeneous, is to examine the trajectories of the Brownian motion of individual probe particles once gelation has taken place. If different probe particle trajectories are similar in terms of their range and their general appear- 


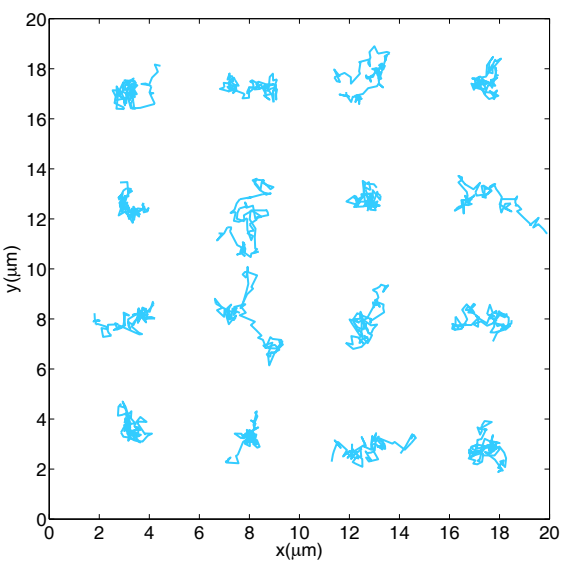

(a)

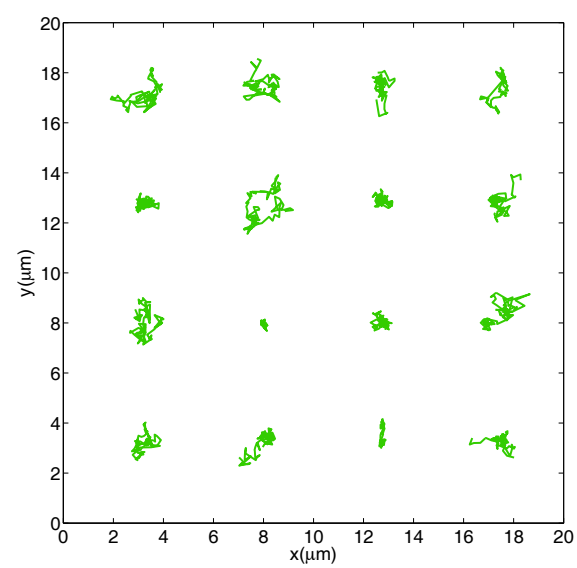

(b)

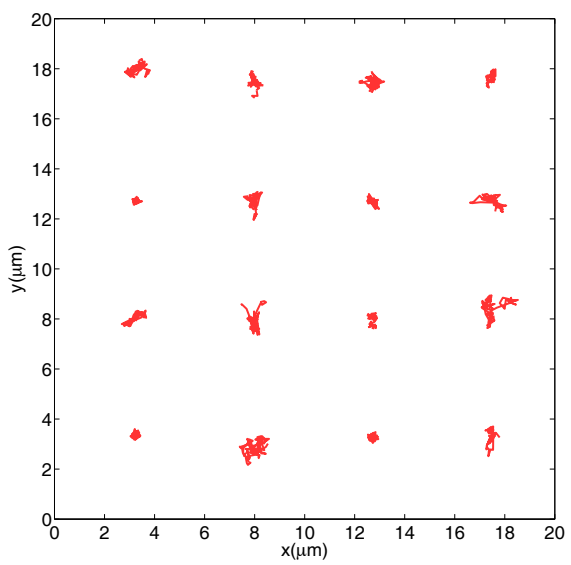

(c)

Fig. 6. Representative probe particle trajectories for hydrogels prepared using $10 \mathrm{mM}$ Fmoc-Y and gelled using GdL concentrations of $5 \mathrm{mM}$ (a), $7.5 \mathrm{mM}$ (b) and $10 \mathrm{mM}$ (c) at $t_{w}=3$ hours. In all three samples, the probe particles appear to diffuse around fixed locations. The range of the particles appears to decrease with increasing GdL concentration. There is a lack of uniformity in the trajectories of particles for all three samples indicating that the samples are heterogeneous.

ance, it indicates that they each probe micro-environments with similar local rheological properties and that the sample is homogeneous. In contrast, if the probe particle trajectories of a sample at a given incubation time, $t_{w}$, are substantially different, indicates that they each probe micro-environments with differing local rheological properties [19]. Given the number of particles being tracked in each measurement it is not feasible to examine all particle trajectories. Rather a representative sample of particle trajectories for each sample at $t_{w}=3 \mathrm{~h}$ is plotted as shown in fig. 6. To ensure comparability between individual probe particle trajectories, all plotted trajectories represent the diffusion of a randomly selected particle over a period of $3 \mathrm{~s}$. This time scale is selected as a trade-off between the need to maximise the duration of the probe particle trajectory and not biasing the results in favour of probe particles which persist for long time scales.

Figure 6 shows that, at late $t_{w}$, the majority of probe particles appear to diffuse around fixed positions. The confinement of a majority of the particles confirms that a gel has indeed formed, although a minority follow tortuous trajectories, an indication that they may be in exploring pores in the gel microstructure. The approximate uniform movement of particles with respect to time means that particles do not "cage-hop" between micro-environments and, therefore, that the heterogeneity being measured is spatial rather than temporal [50]. The overall spatial ranges of the particle trajectories appear to decrease with increasing GdL concentration, consistent with the calculations in sect. 3.2 that show that higher GdL concentrations result in the formation of more robust hydrogels.

Any spatial heterogeneity present in the sample can be examined more quantitatively through a statistical analysis of the distribution of the magnitudes of the MSD at a given lag time. One such statistical approach is to calculate the bin distribution of the particle MSDs. This function is denoted $f(z)[31]$ and is defined as the ratio between the ensemble-averaged MSD of the fraction of $N$ particles, $z$, that have the highest MSD and the ensembleaveraged MSD of all particles

$$
f(z)=\frac{\sum_{i=N(1-z)}^{i=N} \mathrm{MSD}_{i}}{\sum_{i=1}^{i=N} \mathrm{MSD}_{i}} .
$$

For a perfectly homogeneous system, all MSDs are equal and $f(z)=z$. By contrast, if the system is heterogeneous, the highest MSDs have a larger effect on the sum and $f(z)>z$. Thus, the metric $[f(z)-z]$ is a measure of heterogeneity.

The function $f(z)$ is evaluated at $\tau=0.1 \mathrm{~s}$. This low lag time is chosen because the length of particle trajectories is finite and variable and data from short lag times represents a larger number of data points within single particle trajectories as well as a more representative sample of particle trajectories. Hence, sampling the particle displacements at $\tau=0.1 \mathrm{~s}$ ensures that the statistical quality of the function is maximised. Values of $z$ are selected as 10\%, 25\% and 50\% and the analysis is applied to hydrogels prepared using $10 \mathrm{mM}$ Fmoc-Y and gelled using GdL concentrations of $5 \mathrm{mM}, 7.5 \mathrm{mM}$ and $10 \mathrm{mM}$. The resulting values of $[f(z)-z]$ with respect to $t_{w}$ are shown in fig. 7 .

Figure 7 shows $[f(z)-z]$ to deviate from 0 for each value of $z$ used, for each sample studied and over all values of $t_{w}$. To some extent this is not surprising as the effect of poor statistics in the recorded MSD can cause apparent heterogeneity [50]. However at the values of $t_{w}$ at which self-assembly is expected to begin $\left(t_{w} \approx 55\right.$ minutes, 45 minutes and 20 minutes for $5 \mathrm{mM}, 7.5 \mathrm{mM}, 10 \mathrm{mM}$ GdL) the value of $[f(z)-z]$ decreases indicating an apparent decrease in heterogeneity. This is unexpected, as it could be expected that the formation of pre-gel aggregates at 


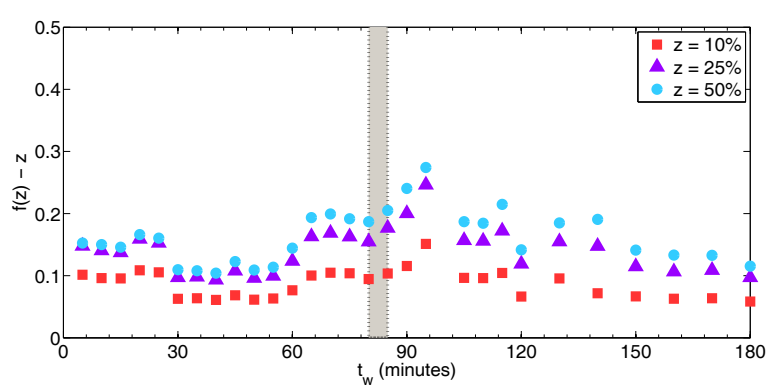

(a)

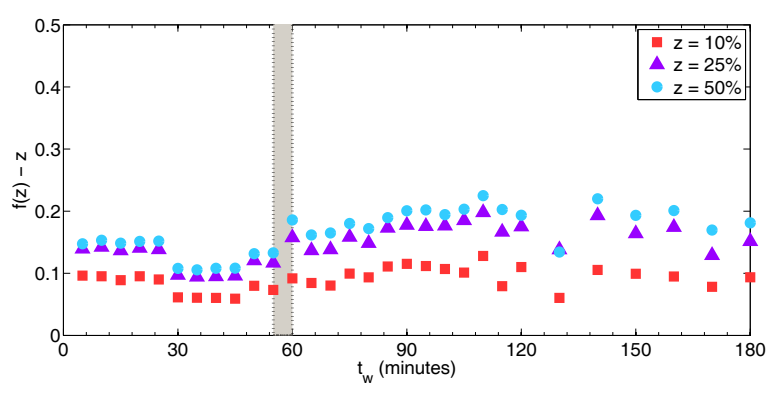

(b)

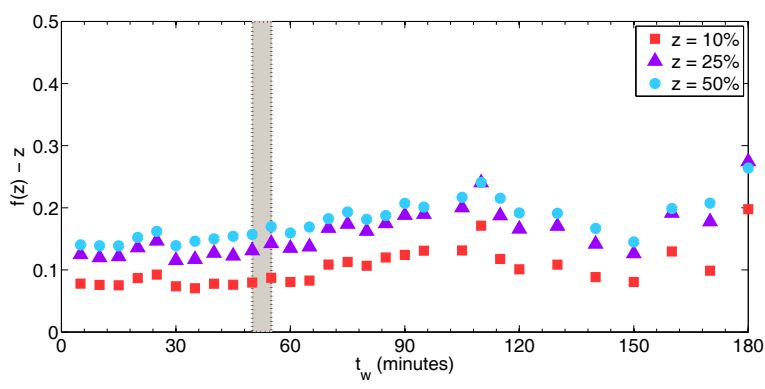

(c)

Fig. 7. Values of the function $[f(z)-z]$ with respect to $t_{w}$ for samples prepared using $10 \mathrm{mM}$ Fmoc-Y and gelled using GdL concentrations of $5 \mathrm{mM}$ (a), $7.5 \mathrm{mM}$ (b) and $10 \mathrm{mM}$ (c). A value of $[f(z)-z]>0$ indicates the presence of heterogeneity in the hydrogel network. At very early $t_{w},[f(z)-z]$ is unexpectedly high, due to errors caused by the high diffusivity of the embedded particles (see text for further explanation). Around the gel point (shown as a shaded region) heterogeneity increases for all values of $z$, with particularly sharp increases for lower GdL concentrations. Heterogeneity at the latest value of $t_{w}$ is higher for higher GdL concentrations.

this time point should cause an increase in heterogeneity. A possible explanation is that the heterogeneity before the onset of self-assembly is shown as artificially higher because the particles are unconstrained in the purely viscous sample and therefore have a long and variable mean free path. Particles in a purely viscous medium can also be expected to diffuse in and out of the field of view at

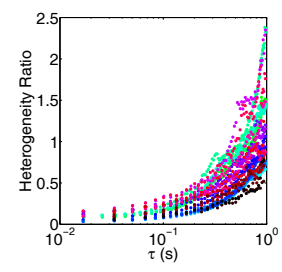

(a)

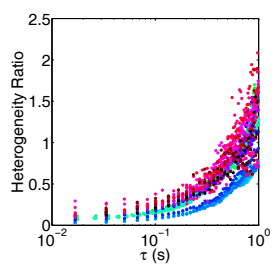

(b)

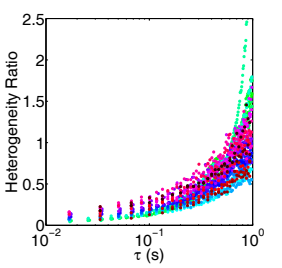

(c)
Fig. 8. Selected plots of the heterogeneity ratio, HR vs. lag time, $\tau$ for samples prepared using $10 \mathrm{mM}$ Fmoc-Y and gelled using GdL concentrations of $5 \mathrm{mM}$ (a), $7.5 \mathrm{mM}$ (b) and $10 \mathrm{mM}$ (c). The different colours indicate different incubation times $t_{w}$. In each of the samples HR is invariant with $\tau$ at low values of $\tau$ but increases rapidly at higher values of $\tau$. This is due to a fundamental limit in values of $\tau$ for which $\mathrm{HR}$ is valid. From examination of plots it can be estimated that $\tau=0.1 \mathrm{~s}$ is within this limit.

fast rates and therefore comparatively little information about their individual displacements is available.

Over later time scales the change in $[f(z)-z]$ with $t_{w}$ is slight and highly variable, giving little indication of how the heterogeneity of the gel changes with time. Broadly speaking, however, it can be said that there is clear increase in $[f(z)-z]$ around the gel point (shown as a shaded region in fig. 7) that is gradual for higher GdL concentrations and more pronounced for lower GdL concentrations, suggesting that for lower GdL concentrations, at the gel point, the gel network is not fully interconnected and that particles exist in a range of different micro-environments. At the longest values of $t_{w}$ (3 hours), the value of $[f(z)-z]$ is higher for higher $\mathrm{GdL}$ concentrations indicating that, although lower $\mathrm{GdL}$ concentrations cause more variation in heterogeneity at intermediate times, the eventual heterogeneity increases with higher GdL concentrations. The value of $[f(z)-z]$ although indicative of heterogeneity is simply a measure of the distribution of MSD values, which is a relative measure that is dependent on a number of external factors such as the number of particles and the depth of tracking of the microscope. As a result it is a metric which has no experimental benchmarks, making it difficult to determine objectively how the values discussed above compare to other systems.

A more rigourous way to measure sample heterogeneity is to use the so-called heterogeneity ratio, abbreviated "HR" [33]. When sampling particle trajectories, the ensemble MSD is biased towards more mobile particles, due to the fact that such particles frequently diffuse through the vertical region that the microscope is able to accurately track. This often results in a large number of short mobile particle trajectories and a smaller number of long immobile particle trajectories, particularly in samples with high levels of heterogeneity. Weighting each particle trajectory by a factor proportional to its length in time, corrects this depth of tracking error and allows an unbiased measure of ensemble average MSD and its variance to be calculated [33]. HR is a dimensionless number and is defined as the ratio between the square of the 


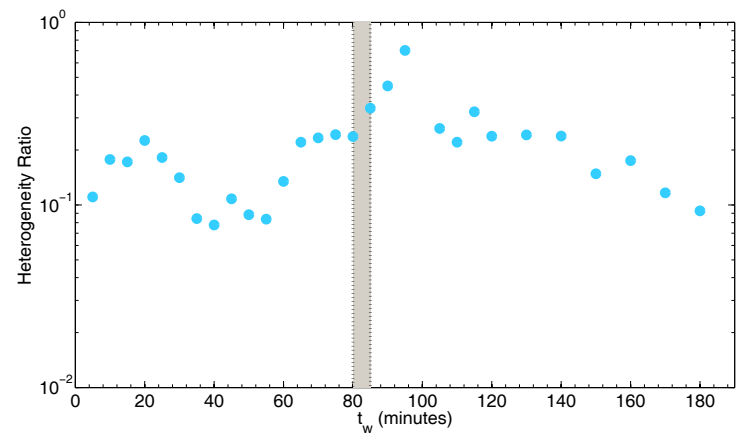

(a)

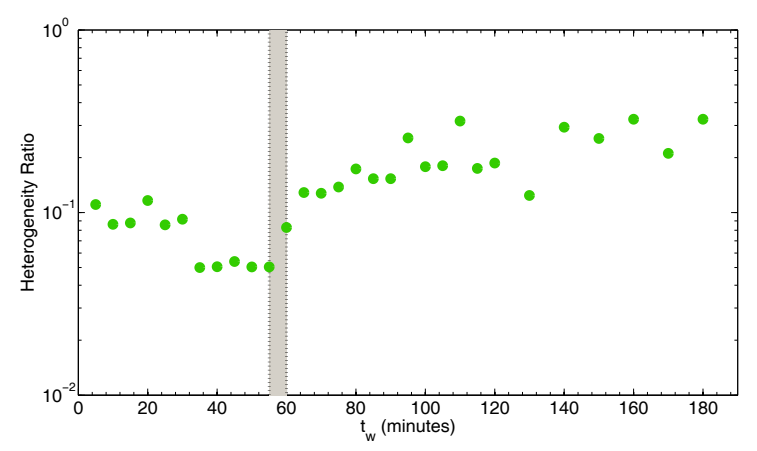

(b)

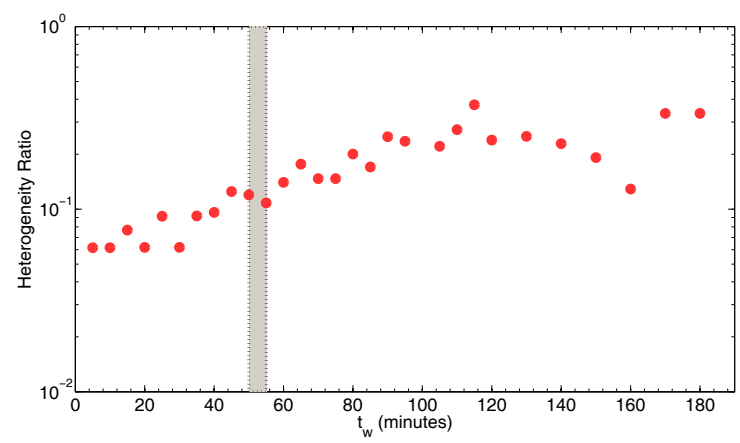

(c)

Fig. 9. Values of the heterogeneity ratio, HR for $\tau=0.1 \mathrm{~s}$ with respect to $t_{w}$ for samples prepared using $10 \mathrm{mM}$ Fmoc-Y and gelled using GdL concentrations of $5 \mathrm{mM}$ (a), $7.5 \mathrm{mM}$ (b) and $10 \mathrm{mM}$ (c). The approximate gel point for each of the samples is shown as a shaded region. HR increases over the range of $t_{w}$ shown for each of the samples but remains comparatively low over all values of $t_{w}$. As with the previously discussed metrics, $[f(z)-z]$ and $N$, for lower concentrations of GdL, fluctuations in HR are apparent immediately after the gel point.

unbiased ensemble MSD $M_{1}(t)$ and its corresponding variance, $M_{2}(t)$ :

$$
\mathrm{HR}=\frac{M_{2}(\tau)}{M_{1}(\tau)^{2}}
$$

There are, however, fundamental limits to the lag times at which $\mathrm{HR}$ is valid [51]. At particularly large lag times, the most diffusive particles will undergo diffusion over dis- tances close to the depth of tracking of the microscope. At such large values of $\tau$, very little data can be extracted from the shortest particle tracks and discrepancies between the biased and unbiased MSD are amplified. This can be seen in fig. 8 where HR is shown to be approximately invariant with $\tau$ for low values of $\tau$ but increases rapidly at higher values of $\tau$. Thus there is an upper maximum value of $\tau$ for which reliable data can be used. From examination of the plots of HR vs. $\tau$ for each of the samples shown in fig. 8, a value of $\tau=0.1 \mathrm{~s}$ is found to be an acceptable cutoff for the vast majority of data sets. The mean HR for $\tau=0.1$ is plotted with respect to $t_{w}$ in fig. 9 .

Figure 9 shows that, as with plots of $[f(z)-z] v s . t_{w}$ (fig. 7), the apparent heterogeneity as measured by HR is unexpectedly high at early $t_{w}$. As before this can be explained by high diffusivity errors.

The longer term trends in HR are also similar to those in $[f(z)-z]$. Heterogeneity appears to increase around the gel point (shown as a shaded region in fig. 9) for each of the three samples. As with $[f(z)-z]$, there is also significant variation between consecutive measurements of values of HR over the values of $t_{w}$ shown. Finally the value of HR at $t_{w}=3$ hours is also dependent on GdL concentration with values of $0.0928,0.325$ and 0.335 for $5 \mathrm{mM}, 7.5 \mathrm{mM}$ and $10 \mathrm{mM}$, respectively.

For comparison, theoretical simulations [33] have indicated that, for a bimodal fluid comprising two component liquids with each liquid making up half the total volume, the maximum possible value of HR is 3 . The same paper showed that a bimodal fluid comprising $50 \%$ water (viscosity $0.9 \mathrm{mPas}$ ) and $50 \%$ Newtonian fluid with viscosity $9 \mathrm{mPas}$ has an HR of 0.6. When seen in this theoretical context the values of $\mathrm{HR}$ at $t_{w}=3$ hours suggest that the hydrogel networks formed in each of the three samples are not particularly heterogeneous. For an experimental comparison, the clay Laponite at a concentration of $1 \%$ by weight was found to have a value of $\mathrm{HR}$ of approximately 1 [19], thereby further confirming that the hydrogels studied here are relatively homogeneous.

A third method of measuring heterogeneity is to examine the distribution of particle displacements in an ensemble of particles at a given lag time. Because particle trajectories are random walks, such a distribution is Gaussian if the particles are probing identical micro-environments. The probability $P$ that at lag time $\tau$ a single particle is displaced by a distance $\Delta x(\tau)$ is therefore given by a Gaussian probability distribution

$$
P(\Delta x, \tau)=(4 \pi D \tau)^{-\frac{1}{2}} \exp \left(-\frac{\Delta x(\tau)^{2}}{4 D \tau}\right)
$$

where $D$ is the diffusion coefficient. Therefore heterogeneity in the sample at the length scale being probed is manifested as a deviation from Gaussian behaviour in the particle displacement probability density function, commonly referred to as the van Hove correlation function. The extent to which this deviation occurs is defined by the nonGaussian parameter $N$, the ratio between the fourth moment and the square of the second moment of a best fit 


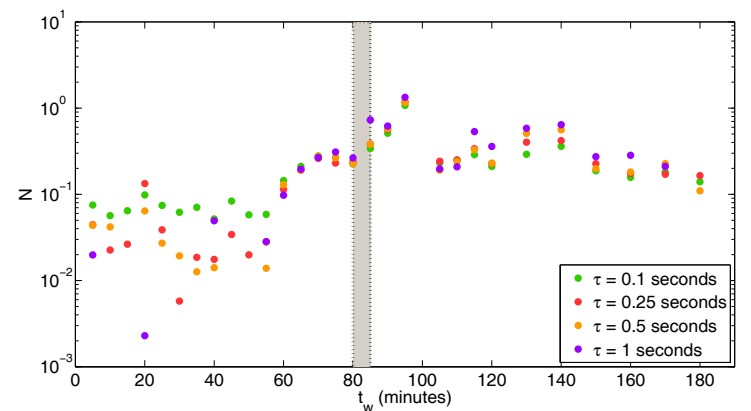

(a)

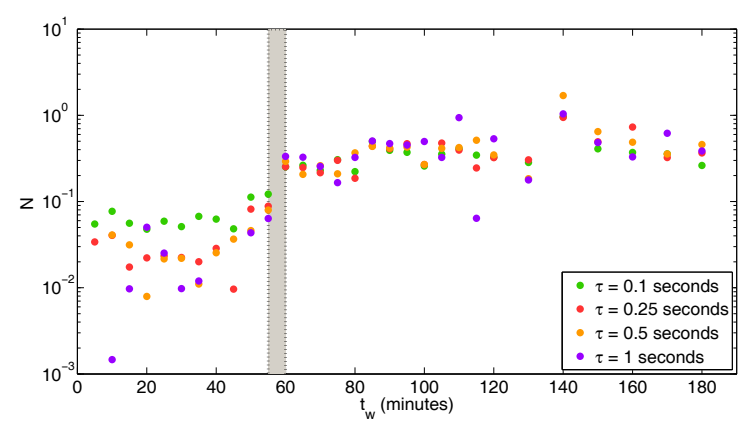

(b)

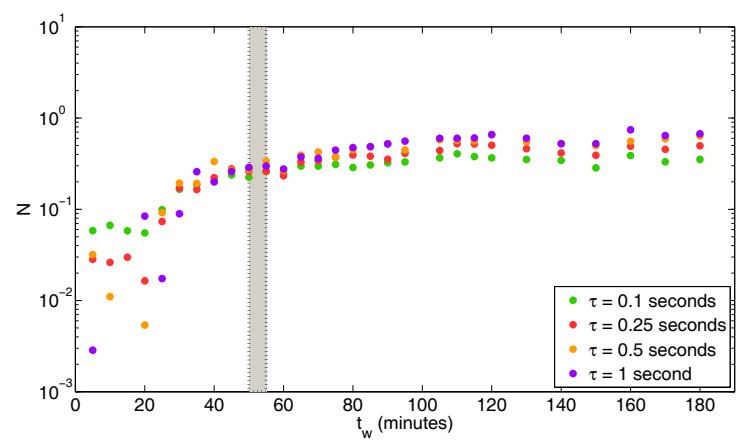

(c)

Fig. 10. Values of the non-Gaussian parameter, $N$ for $\tau=0.1 \mathrm{~s}$ with respect to $t_{w}$ for samples prepared using $10 \mathrm{mM}$ Fmoc$\mathrm{Y}$ and gelled using GdL concentrations of $5 \mathrm{mM}$ (a), $7.5 \mathrm{mM}$ (b) and $10 \mathrm{mM}$ (c). The approximate gel point for each of the samples is shown as a shaded region. $N$ increases over the range of $t_{w}$ shown for each of the samples but remains comparatively low over all values of $t_{w}$ (see text further discussion). Values of $N$ appear to be dependent on $\tau$, indicating that over the longer length scales probed at these longer values of $\tau$ the heterogeneity is higher.

to the van Hove function [32]:

$$
N=\frac{\left\langle x^{4}\right\rangle}{\left\langle 3 x^{2}\right\rangle^{2}}-1
$$

In a perfectly homogenous sample, $N=0$, representing a perfect Gaussian fit. Higher values of $N$ indicate the presence of heterogeneity.

Because $N$ is calculated from individual particle displacements, rather than mean-squared displacements (as is the case with $[f(z)-z]$ and HR) the number of data points used to calculate each value of $N$ is higher. Thus using $N$ as opposed to $[f(z)-z]$ and HR gives improved statistics. This means $N$ can be evaluated over a wider range of lag times, without compromising the statistics. Calculated values of $N$ with respect to incubation time $t_{w}$ are shown in fig. 10. $N$ is evaluated at a range of values of $\tau$ and the van Hove correlation function is calculated from averaging the particle displacements in the $x$ and $y$ dimension to improve statistics [32].

The evolution in $N$ in fig. 10 can be said to be qualitatively similar to evolution in $[f(z)-z]$ and HR in figs. 7 and 9 , respectively. However, the increase in $N$ with $t_{w}$ appears to be smoother. Variations in consecutive measurements are present but are much less severe than those for $[f(z)-z]$ and HR. Another notable difference between the heterogeneity indicated by values of $N$ and that from values of $[f(z)-z]$ and $\mathrm{HR}$ is that the apparent heterogeneity at very early $t_{w}$ is relatively low. This suggests that the metric $N$ is less prone to high diffusivity errors than either of the other two metrics examined. This is unsurprising given that the high diffusivity error is predicted to arise from the time- and ensemble-averaging used to calculate the MSD. The metrics $[f(z)-z]$ and HR are both calculated by analysing the ensemble- and time-averaged MSD, whereas $N$ is calculated directly from particle displacement data. It can therefore be surmised that overall $N$ is a more reliable metric for quantifying the heterogeneity of the samples studied here. The relative limitations of $[f(z)-z]$ and HR may have implications for other studies of heterogeneity because, to the best knowledge of the authors, the three metrics have not previously been compared in this way.

A further distinction between the metrics is that the value of $N$ appears to be dependent on lag time $\tau$. This is most apparent in fig. 10(c) where $N$ clearly increases with increasing lag time. The apparent heterogeneity therefore increases with $\tau$, indicating that over the longer length scales probed at these longer values of $\tau$ the heterogeneity is higher.

Values of $N$ at $t_{w}=3$ hours are clearly dependent on GdL concentration with values of $0.221,0.262$ and 0.352 for $5 \mathrm{mM}, 7.5 \mathrm{mM}$ and $10 \mathrm{mM}$, respectively. Comparing these metrics with Laponite at a concentration of $1 \%$ by weight $(N=10[18])$ further confirms that the hydrogels studied here are relatively homogeneous, in line with the data from $[f(z)-z]$ and HR.

It should be noted that, for each of the samples studied here, none of the three metrics used reports a significant rise in heterogeneity after the gel point. This is in direct contrast to data systems that are more heterogeneous, where heterogeneity is observed to continue rising after the gel point [18]. It should also be noted that due to the evolving nature of the hydrogel, it is not possible to study heterogeneity at particularly long lag times as individual microrheology measurements must be short enough to ensure that the sample does not change significantly during measurement. 


\section{Summary}

In this work particle tracking microrheology has been shown to be a valid and effective technique in measuring the dynamic rheological and structural properties of the self-assembling peptide derivative Fmoc-Y. The use of Glucono- $\delta$-Lactone as a gelation agent ensures a gradual gelation process, which can be monitored in real time.

Comparisons between plots of $p \mathrm{H}$ vs. incubation time (fig. 2) and probe particle MSD vs. incubation time (fig. 4) show that the characteristic rise in $p \mathrm{H}$ close to the $p K_{a}$ correlates approximately with a drop in MSD (signifying an increase in viscosity), a phenomenon that has been noted in previous studies by the authors under similar conditions [45]. This study has shown the same to be true over a range of different GdL concentrations.

At later incubation times particle tracking data shows that each of the samples form hydrogels at time scales that decrease with increasing GdL concentration. MSD at $t_{w}=3$ hours decreases with increasing GdL concentration, suggesting that gel strength is lower at a given time when lower GdL concentrations are used. For lower GdL concentrations the measured MSD of the sample appears to fluctuate between gel-like and liquid-like properties, a phenomenon tentatively attributed to the possible breaking and re-forming of bonds between aggregates in the hydrogel network. The authors also postulate that this fluctuation may account for some of the variability in final hydrogel properties recently reported in similar systems [49].

The spatial heterogeneity in the hydrogel network with respect to $t_{w}$ is studied through the application of three quantitative metrics: the heterogeneity ratio (HR), the non-Gaussian parameter of the van Hove correlation function $(N)$ and the bin distribution of the single particle MSD $(f(z))$. Results between the three metrics are found to be approximately comparable although $\mathrm{HR}$ and $f(z)$ are found to exhibit an additive error at early incubation times due to limited statistics for individual particles, as the particles have a short mean free path. Measured values of HR and $f(z)$ are also shown to exhibit significantly more variation over consecutive measurements than values of $N$. It can therefore be surmised that, for the samples examined in this study, $N$ is an overall more reliable metric for quantifying the heterogeneity. This is likely to be due to the fact that $N$ is calculated from individual particle displacements, rather than mean-squared displacements (as is the case with $[f(z)-z]$ and HR) and the number of data points used to calculate each value of $N$ is higher. Thus using $N$ as opposed to $[f(z)-z]$ and HR allows heterogeneity to be evaluated with respect to $\tau$ due to improved statistics.

Plots of $N$ vs. $t_{w}$ also show that heterogeneity appears to change over different lag times and therefore over different length scales, a finding that opens up the possibility for further work, for example through 2-particle microrheology [52] or through using a variety of probe particles with different diameters [19].

Of the three metrics, two of them, $\mathrm{HR}$ and $N$, can be said to be both quantitative and absolute (i.e. comparable across different experiments and different materials). The values of $\mathrm{HR}$ and $N$ are in the range: $\mathrm{HR}=0.0928-0.335$; and $N=0.221-0.352$ for the range of GdL concentrations used at $t_{w}=3$ hours. These values are smaller than those previously reported in other systems. This is unremarkable, given that neither of these metrics have been used extensively or for systems which are not known to be highly heterogeneous. Contextualising the values of HR and $N$ by applying them to a wider range of materials in future work would therefore be beneficial, bearing in mind the limits to their statistical validity as demonstrated in this study.

This work formed part of the doctoral thesis of A.A-R. The authors gratefully acknowledge funding from the EPSRC and Unilever R\&D through an EPSRC CASE studentship [34].

Open Access This is an open access article distributed under the terms of the Creative Commons Attribution License (http://creativecommons.org/licenses/by/4.0), which permits unrestricted use, distribution, and reproduction in any medium, provided the original work is properly cited.

\section{References}

1. John B. Matson, R. Helen Zha, Samuel I. Stupp, Curr. Opin. Solid State Mater. Sci. 15, 225 (2011).

2. Shuguang Zhang, Fabrizio Gelain, Xiaojun Zhao, Semin. Cancer Biol. 15, 413 (2005).

3. Dennis E. Discher, Adi Eisenberg, Science 297, 967 (2002).

4. Lara A. Estroff, Andrew D. Hamilton, Chem. Rev. 104, 1201 (2004).

5. Jaclyn Raeburn, Andre Zamith Cardoso, Dave J. Adams, Chem. Soc. Rev. 42, 5143 (2013).

6. Maaike de Loos, Ben L. Feringa, Jan H. van Esch, Eur. J. Org. Chem. 2005, 3615 (2005).

7. Joel P. Schneider, Darrin J. Pochan, Bulent Ozbas, Karthikan Rajagopal, Lisa Pakstis, Juliana Kretsinger, J. Am. Chem. Soc. 124, 15030 (2002).

8. Jeffrey D. Hartgerink, Elia Beniash, Samuel I. Stupp, Science 294, 1684 (2001).

9. Maya J. Pandya, Gillian M. Spooner, Margaret Sunde, Julian R. Thorpe, Alison Rodger, Derek N. Woolfson, Biochemistry 39, 8728 (2000).

10. Yan Zhang, Zhimou Yang, Fang Yuan, Hongwei Gu, Ping Gao, Bing Xu, J. Am. Chem. Soc. 126, 15028 (2004).

11. Zhimou Yang, Gaolin Liang, Manlung Ma, Yuan Gao, Bing Xu, J. Mater. Chem. 17, 850 (2007).

12. Dave J. Adams, Paul D. Topham, Soft Matter 6, 3707 (2010).

13. Z. Yang, H. Gu, D. Fu, P. Gao, J.K. Lam, B. Xu, Adv. Mater. 16, 1440 (2004).

14. Zhimou Yang, Hongwei Gu, Yan Zhang, Ling Wang, Bing $\mathrm{Xu}$, Chem. Commun., issue 2, 208 (2004).

15. Z.A.C. Schnepp, R. Gonzalez-McQuire, S. Mann, Adv. Mater. 18, 1869 (2006).

16. Sam Sutton, Neil L. Campbell, Andrew I. Cooper, Mark Kirkland, William J. Frith, Dave J. Adams, Langmuir 25, 10285 (2009). 
17. Dave J. Adams, Michael F. Butler, William J. Frith, Mark Kirkland, Leanne Mullen, Paul Sanderson, Soft Matter 5, 1856 (2009).

18. Felix K. Oppong, P. Coussot, John R. de Bruyn, Phys. Rev. E 78, 021405 (2008).

19. Jason P. Rich, Gareth H. McKinley, Patrick S. Doyle, J. Rheol. 55, 273 (2011).

20. Kevin Channon, Cait E. MacPhee, Soft Matter 4, 647 (2008).

21. Celeste M. Nelson, Joe Tien, Curr. Opin. Biotechnol. 17, 518 (2006).

22. Anita C. Bellail, Stephen B. Hunter, Daniel J. Brat, Chalet Tan, Erwin G. Van Meir, Int. J. Biochem. Cell Biol. 36, 1046 (2004).

23. Philip S. Stewart, Michael J. Franklin, Nat. Rev. Micro 6, 199 (2008).

24. Thomas G. Mason, D.A. Weitz, Phys. Rev. Lett. 74, 1250 (1995).

25. A.M. Corrigan, A.M. Donald, Eur. Phys. J. E 28, 457 (2009).

26. Thomas A. Waigh, Rep. Prog. Phys. 68, 685 (2005).

27. Joshua Apgar, Yiider Tseng, Elena Fedorov, Matthew B. Herwig, Steve C. Almo, Denis Wirtz, Biophys. J. 79, 1095 (2000).

28. Yiider Tseng, Denis Wirtz, Biophys. J. 81, 1643 (2001).

29. Alan Goodman, Yiider Tseng, Denis Wirtz, J. Mol. Biol. 323, 199 (2002).

30. Jingyuan Xu, Yiider Tseng, Craig J. Carriere, Denis Wirtz, Biomacromolecules 3, 92 (2002).

31. Yiider Tseng, Kwang M. An, Denis Wirtz, J. Biol. Chem. 277, 18143 (2002).

32. Willem K. Kegel, Alfons van Blaaderen, Science 287, 290 (2000).

33. Thierry Savin, Patrick S. Doyle, Phys. Rev. E 76, 021501 (2007).

34. Anders Aufderhorst-Roberts, Microrheological Characterisation of Fmoc Derivative Hydrogels, $\mathrm{PhD}$ thesis, Wolfson College, University of Cambridge (2012).

35. Dave J. Adams, Leanne M. Mullen, Marco Berta, Lin Chen, William J. Frith, Soft Matter 6, 1971 (2010).
36. P.J. Kocienski, Protecting Groups (Foundations of Organic Chemistry) (Thieme Publishing Group, 1994).

37. Y. Pocker, Edmond. Green, J. Am. Chem. Soc. 95, 113 (1973).

38. Adam M. Corrigan, Particle Tracking Microrheology of Fibrillar Protein Networks, PhD thesis, Darwin College, University of Cambridge (2009).

39. Imran A. Hasnain, Athene M. Donald, Phys. Rev. E 73, 031901 (2006).

40. D. Marr, E. Hildreth, Theory of Edge Detection, Vol. 207 (The Royal Society, 1980).

41. M. Atakhorrami, J.I. Sulkowska, K.M. Addas, G.H. Koenderink, J.X. Tang, A.J. Levine, Phys. Rev. E 73, 061501 (2006).

42. Thierry Savin, Patrick S. Doyle, Biophys. J. 88, 623 (2005).

43. A. Papagiannopoulos, T.A. Waigh, T.E. Hardingham, Faraday Disc. 139, 337 (2008).

44. Claire Tang, Andrew M. Smith, Richard F. Collins, Rein V. Ulijn, Alberto Saiani, Langmuir 25, 9447 (2009).

45. Anders Aufderhorst-Roberts, William J. Frith, Athene M. Donald, Soft Matter 8, 5940 (2012).

46. H. Henning Winter, Francois Chambon, J. Rheol. 30, 367 (1986).

47. T.G. Mason, K. Ganesan, J.H. Van Zanten, D. Wirtz, S.C. Kuo, Phys. Rev. Lett. 79, 3282 (1997).

48. Andre Zamith Cardoso, Ana Estefania Alvarez Alvarez, Beatrice N. Cattoz, Peter C. Griffiths, Stephen M. King, William J. Frith, Dave J. Adams, Faraday Disc. 166, 101 (2013).

49. Wilda Helen, Piero de Leonardis, Rein V. Ulijn, Julie Gough, Nicola Tirelli, Soft Matter 7, 1732 (2011).

50. Megan T. Valentine, Peter D. Kaplan, D. Thota, John C. Crocker, Thomas Gisler, Robert K. Prud'homme, M. Beck, David A. Weitz, Phys. Rev. E 64, 061506 (2001).

51. Thierry Savin, Microrheology of heterogeneous systems, online tutorial http://web.mit.edu/savin/Public/.Tutorial_v1.2/ (2008).

52. Alex. J. Levine, T.C. Lubensky, Phys. Rev. Lett. 85, 1774 (2000). 